\title{
The Long-Term Footprint of Endometriosis : Population-Based Cohort Analysis Reveals Increased Pain Symptoms and Decreased Pain Tolerance at Age 46 Years
}

\section{Vuontisjarvi, Saara}

2018-07

Vuontisjarvi , S , Rossi , H-R, Herrala , S, Morin-Papunen, L, Tapanainen, J S , Karjula , S , Karppinen , J , Auvinen , J \& Piltonen , T T 2018 , ' The Long-Term Footprint of Endometriosis : Population-Based Cohort Analysis Reveals Increased Pain Symptoms and Decreased Pain Tolerance at Age 46 Years ' , Journal of Pain , vol. 19 , no. 7 , pp. 754-763 . https://doi.org/10.1016/

http://hdl.handle.net/10138/299818

https://doi.org/10.1016/j.jpain.2018.02.005

cc_by_nc_nd

acceptedVersion

Downloaded from Helda, University of Helsinki institutional repository.

This is an electronic reprint of the original article.

This reprint may differ from the original in pagination and typographic detail.

Please cite the original version. 


\section{Accepted Manuscript}

Title: The Long-Term Footprint of Endometriosis: Population-Based Cohort Analysis Reveals Increased Pain Symptoms and Decreased Pain Tolerance at Age 46

Author: Saara Vuontisjärvi, Henna-Riikka Rossi, Sauli Herrala, Laure MorinPapunen, Juha S. Tapanainen, Salla Karjula, Jaro Karppinen, Juha Auvinen, Terhi T. Piltonen

PII: S1526-5900(18)30085-3

DOI: https://doi.org/10.1016/j.jpain.2018.02.005

Reference: YJPAI 3536

To appear in: The Journal of Pain

Received date: $\quad 9-11-2017$

Revised date: $\quad 2-2-2018$

Accepted date: $14-2-2018$

Please cite this article as: Saara Vuontisjärvi, Henna-Riikka Rossi, Sauli Herrala, Laure MorinPapunen, Juha S. Tapanainen, Salla Karjula, Jaro Karppinen, Juha Auvinen, Terhi T. Piltonen, The Long-Term Footprint of Endometriosis: Population-Based Cohort Analysis Reveals Increased Pain Symptoms and Decreased Pain Tolerance at Age 46, The Journal of Pain (2018), https://doi.org/10.1016/j.jpain.2018.02.005.

This is a PDF file of an unedited manuscript that has been accepted for publication. As a service to our customers we are providing this early version of the manuscript. The manuscript will undergo copyediting, typesetting, and review of the resulting proof before it is published in its final form. Please note that during the production process errors may be discovered which could affect the content, and all legal disclaimers that apply to the journal pertain. 
1 The Long-term Footprint of Endometriosis: Population-based Cohort Analysis Reveals Increased Pain Symptoms and Decreased Pain Tolerance at Age 46

Saara Vuontisjärvi ${ }^{1,4}$, Henna-Riikka Rossi ${ }^{1,4}$, Sauli Herrala², Laure Morin-Papunen ${ }^{1,4}$, Juha S. Tapanainen $^{1,3,4}$, Salla Karjula ${ }^{1,4}$, Jaro Karppinen ${ }^{2,4,5}$, Juha Auvinen ${ }^{2,4}$, Terhi T. Piltonen ${ }^{1,4,6^{*}}$

${ }^{1}$ Department of Obstetrics and Gynecology, Oulu University Hospital, University of Oulu and PEDEGO Research Unit, Oulu, Finland

9 P.O.Box 23, Fl-90029 Oulu University Hospital, Finland

$10 \quad{ }^{2}$ Center for Life Course Health Research, University of Oulu, Oulu, Finland

11 P.O.Box 8000, FI-90014 University of Oulu, Finland

$12{ }^{3}$ Department of Obstetrics and Gynecology, Helsinki University Hospital, University of Helsinki,

13 Helsinki, Finland

14 P.O.Box 63, Biomedicum Helsinki, 00014 University of Helsinki, Finland

$15{ }^{4}$ Medical Research Center Oulu, Oulu University Hospital and University of Oulu, Oulu, Finland

16 P.O.Box 8000, Fl-90014 University of Oulu, Finland

$17 \quad{ }^{5}$ Finnish Institute of Occupational Health, Oulu, Finland

18 P.O.Box 40, FI-00251, Helsinki, Finland

$19{ }^{6}$ Corresponding author

$21 \quad{ }^{6}$ Terhi T. Piltonen M.D., Ph.D. Associate Professor

22 Consultant, Clinical Researcher for the Finnish Medical Foundation

23 Department of Obstetrics and Gynecology

24 PEDEGO Research Unit, Medical Research Center

25 Oulu University Hospital, University of Oulu

26 Kajaanintie 50, BOX 5000, 90014 Oulu, FINLAND

27 terhi.piltonen@oulu.fi 
28 Phone: +358 83153051 , Mobile: +358 405008266

29 The preliminary data were presented as an oral presentation at the ESHRE 07/2016 meeting in

30 Helsinki, Finland.

31 Keywords: Endometriosis, Pain Threshold, Pain Tolerance, Pain Troublesomeness 32

33 Disclosures:

34 The authors declare no conflicts of interest.

35 The study was funded by The Academy of Finland (project grants 104781, 120315, 129269,

36 1114194, 268336, SALVE), the Sigrid Jusélius Foundation, The Finnish Medical Foundation, the

37 North Ostrabothnia Regional Fund, Northern Finland Health Care Support Foundation, University

38 Hospital Oulu, Biocenter, University of Oulu, Finland (75617), the European Commission (EURO-

39 BLCS, Framework 5 award QLG1-CT-2000-01643) and the Medical Research Council, UK

40 (PrevMetSyn/SALVE).

41

42

43 

Highlights

- Endometriosis has been shown to increase pain sensitivity in fertile-aged women.

- The study shows decreasd pain threshold and maximal pain tolerance in women with endometriosis at age 46

- Women with endometriosis report increased pain sites and bothersome and intense pain at age 46

- Delay in diagnosis of endometriosis may lead to increased pain sensitization

- Endometriosis should be diagnosed and treated early on to ensure minimal comorbidity

Abstract

Previous studies have shown increased pain sensitivity in fertile-aged women with endometriosis in response to mechanical stimuli. As yet, population-based studies on the association of endometriosis with pain sensation and pain symptoms in late fertile age are lacking. The main objective of this population-based cohort study was to investigate whether a history of endometriosis is associated with altered pain sensation and musculoskeletal pain symptoms at age 46 .

Our data is derived from the Northern Finland Birth Cohort 1966, which contains postal questionnaire data ( $72 \%$ response rate) as well as clinical data assessing pressure-pain threshold (PPT) and maximal pain tolerance (MaxPTo). The study population consisted of 284 women with endometriosis and 3390 controls.

Our results showed that at age 46 women with a history of endometriosis had a $5.3 \%$ lower PPT and $5.1 \%$ lower maxPTo compared with controls. The most significant contributors besides endometriosis were anxiety, depression and current smoking status. Women with endometriosis also reported an increased number of pain sites (0 pain sites, 9.6 vs. $17.9 \%$; $5-8$ pain sites, 24.8 vs. $19.1 \%$, endometriosis vs. controls respectively, $p<0.001)$, and their pain was more troublesome and intense. The results were adjusted for 
72 BMI, smoking, depressive/anxiety symptoms, education and use of hormonal

73 contraceptives.

74 This unique data revealed an altered pain sensation and a greater likelihood of reporting 75 musculoskeletal pain at age 46 among women with a history of endometriosis. The results 76 imply that endometriosis has a long-term footprint on affected women, thus underlying the 77 need for psychological support and medical treatment beyond fertile age.

78

79

80 Perspective item

81 This is a population-based cohort study showing decreased pain threshold and maximal

82 pain tolerance in women with endometriosis up till late fertile age of 46 years. The pain

83 was also found to be more bothersome and intense compared with controls. 


\section{Introduction}

Endometriosis is an estrogen-dependent, chronic gynecological disorder associated with pelvic pain and infertility, with a prevalence of $6-10 \%$ in the general population $6,7,13$. Affected women experience dysmenorrhea, deep dyspareunia, dyschezia and dysuria ${ }^{13,37}$ associated with low quality of life ${ }^{8}$. The disorder is under-diagnosed or there is a delay in diagnosis in many cases leading to chronic pelvic pain (CPP) ${ }^{7,28}$. Diagnosis is made by laparoscopy or laparotomy, where endometrial lesions are found in extra-uterine locations, mainly the peritoneum and ovaries ${ }^{6,7,13}$. As endometriosis is not curable, its treatments and therapies are targeted at infertility and symptom relief ${ }^{7,11}$. Endometriosis is also associated with other co-morbid conditions such as fibromyalgia and chronic fatigue syndrome ${ }^{11,28}$. Moreover, it has a significant adverse impact on work productivity, social activity, family responsibilities, and daily life, resulting in a substantial economic burden on society ${ }^{11,30}$.

It is well accepted that endometriosis is associated with dysmenorrhea, but it is not known why some women undergo transition to a state of chronic pain, while others do not ${ }^{1}$. Depending on the study population, $30-70 \%$ of women with CPP have laparoscopic evidence of endometriosis ${ }^{21,34}$. The pain symptoms, however, are poorly correlated to the severity of endometriosis, and the pathophysiology of endometriosisassociated pain remains somewhat elusive $9,14,23,25,36,37$. Pain mechanisms in endometriosis are thought to be multifactorial; pain may be nociceptive, neuropathic or a combination of these, and emotional, cognitive and behavioral components are also present $3,20,23,32$. Previous studies have shown increased pain sensitivity among women with endometriosis, with or without CPP, in response to mechanical stimuli compared with control $^{2}$. Furthermore, pain-threshold studies have suggested hyperalgesia at extra-pelvic 
108 sites, most likely due to peripheral and/or central sensitization mechanisms in affected 109 women $5,12,16,23,24$.

Endometriosis is anticipated to subside in menopause, as it is an estrogen-

111 dependent disorder. However, in cases of peripheral and central sensitization, pain

112 symptoms and hyperalgesia may persist beyond fertile age. As yet, no population-based 113 data exist on pain symptoms among women with a history of endometriosis at late 114 reproductive age. Thus, the aim of this study was to determine in a population-based 115 cohort study setting whether women with a self-reported history of endometriosis 116 experience altered pressure-pain sensitivity and adverse pain symptoms at age 46.

118 Materials and Methods

119 Study Population

120 The study population originated from the Northern Finland Birth Cohort 1966 (NFBC1966, 121 http://www.oulu.fi/nfbc) which is a unique, large, prospective, longitudinal dataset 122 comprising all expected births in 1966 in the Northern Finland area (live-born children $123 n=12058$, females $n=5889$ ). Originally the cohort was established to investigate the life124 courses of various health-related conditions. Enrollment in this database began at the $24^{\text {th }}$ 125 gestational week, and, after birth, data-collection points were established at ages 1, 14, 31 126 and 46 years, this study utilizing the latest data-collection point, thus being a secondary, 127 cross-sectional analysis of a prospective study cohort.

At 46 years of age, all participants who were alive and whose postal address 129 in Finland was known received a questionnaire (5123 women). This was the first 130 questionnaire in this longitudinal cohort study including questions on history of 131 endometriosis and pain symptoms. The response rate was $72 \%$. Furthermore, all women 132 were also invited to undergo clinical examination including pressure-pain testing, and 2774 
133 (55\%) participated. All participants gave informed consent. The study followed the

134 principles of the Declaration of Helsinki and the Ethics Committee of the Northern

135 Ostrobothnia Hospital District approved the research. A flow chart of the study is shown in 136 Figure 1.

137 Diagnosis of Endometriosis

138 The final analysis concerned all women self-reporting endometriosis, and those stating "no 139 endometriosis" were considered as controls. Self-reported diagnosis was derived from the 140 postal questionnaire item: "Have you ever been diagnosed with endometriosis by a 141 physician?" resulting in an endometriosis population of 284 women (8\% among women 142 who answered the endometriosis question). There were 3390 women (92\%) reporting no 143 endometriosis and were considered as controls (Figure 1A).

\section{$144 \quad$ Verification of diagnosis}

145 Self-reported diagnosis of endometriosis has only recently been described in the literature $146{ }^{26}$; hence the validity of the diagnosis was verified for the present study through the patient 147 records available at the original study site at Oulu University Hospital (Supplemental 148 Figure). Thirty-seven women (13\%) did not give permission to enter their patient records. 149 Of the 284 women with endometriosis we found patient records for $92(32.4 \%)$. According 150 to the patient records available, 71/92 women (77.2 \%) were diagnosed as having 151 endometriosis, of which $90.1 \%$ were established in laparoscopy/laparotomy. Fifteen 152 women did not have a diagnosis of endometriosis and six were classified as unclear 153 cases. It is possible that the diagnosis was established later in another hospital after 154 moving from the area (groups "no endometriosis" and "unclear cases"). We also estimated 155 the specificity of diagnoses from the national hospital discharge register including 156 diagnosis established during hospital polyclinic visits or during hospitalization. In the 157 endometriosis group $52 \%$ of the women also had a diagnosis in the national hospital 
158 discharge register, compared with $1.5 \%$ among the women reporting not having

159 endometriosis (Table 1). Thus, we concluded that a self-reported history of endometriosis

160 is sufficiently a valid tool to identify endometriosis cases in this cohort.

161 Pressure-Pain Threshold (PPT) and Maximal Pain Tolerance (MaxPTo)

162 Pain measurements were carried out in 2470 controls and 234 women with endometriosis.

163 A few of the four measurement-site readings were missing as a result of technical 164 difficulties. Pressure-pain threshold and maxPTo readings were acquired using an 165 algometer (Somedic AB, Hörby, Sweden) with a 10-mm contact head, which was applied 166 perpendicularly to the skin. Briefly, the pressure was increased from $0 \mathrm{kPa}$ at a constant 167 rate of $50 \mathrm{kPa} / \mathrm{s}$. Instructions to participants were, "A pressure will be applied at a gradual 168 rate. Allow the pressure to increase until it reaches a point where it feels uncomfortable 169 and then press the button down. As we continue increasing the pressure, release the 170 button when you cannot tolerate the pressure any more". The former pressure value was 171 recorded as the PPT and the latter as MaxPTo. Pressure was terminated at the latest 172 when the safety maximum of $1200 \mathrm{kPa}$ was reached. The PPT and MaxPTo 173 measurements were taken at four anatomical sites in the following order: 1) shoulder; the 174 mid-point of the upper trapezius muscle (subject in a prone position), 2) the mid-point of 175 the tibialis anterior muscle (supine position), 3) the dorsal aspect of the wrist joint line 176 (supine position), and 4) the L5/S1 interspinous space (prone position). The test sites were 177 identified and participants were positioned in a standardized manner. Each site was tested 178 twice. Of the peripheral sites, primarily the right side was used. The exact anatomical point 179 of pressure was shifted slightly between the tests in order to prevent sensitization of 180 nociceptors at the contact site. The highest value of the two measurements was used in 181 the analysis to avoid overestimating pain threshold or tolerance. In addition, mean PPT 
182 and MaxPTo values at the four measured locations were calculated and used in the 183 analyses.

184 Questionnaires on pain sites, pain intensity and pain troublesomeness

185 The numbers of musculoskeletal pain sites were assessed as follows: $0,1,2,3,4$ or $5-8$

186 sites. The pain sites were derived from the questionnaire, in which the prevalence of 187 musculoskeletal pain during the previous 12-month period was investigated as follows: 188 "Have you had any aches or pains in the following areas of your body?" 1) neck, 2) 189 shoulders, 3) arms/elbows, 4) wrists/hands/fingers, 5) lower back, 6) hips, 7) knees, and 8) 190 ankles/feet. The anatomical sites were illustrated in a drawing. If there had been pain,

191 there was a following question on the frequency of pain: "How often have you had aches

192 or pains during the last 12 months?" 1) not at all, 2) 1-7 days, 3) 8-30 days, 4) over 30 193 days, or 5) daily. If the person had experienced pain during the past 12 months, pain 194 intensity and pain symptoms at work, during leisure time and sleep, at all musculoskeletal 195 sites, were assessed by using a Numerical Rating Scale (NRS) off 0 (no pain / no 196 disability) to 10 (extremely severe or disabling pain).

\section{Confounders}

198 Infertility

199 Infertility was inquired about at age 46: "Have you ever suffered from infertility (yes/no)?"

200 Parity

201 Parity was inquired about at age 46: "How many deliveries you have experienced?" We 202 divided the women according to parity into three groups: no delivery, one delivery or more 203 than one delivery. 
211 Height and weight were both self-reported and measured at 46 years. In the clinical 212 examinations, participants' weight $(\mathrm{kg})$ was measured with a digital scale, which was 213 calibrated regularly. Height $(\mathrm{cm})$ was measured twice by using a standard calibrated 214 stadiometer. BMI $\left(\mathrm{kg} / \mathrm{m}^{2}\right)$ was calculated by using measured height (average of two 215 measurements) and weight. Self-reported values were used if measured data was not 216 available. There was no statistically significant difference between the self-reported and 217 clinically measured BMI values.

\section{Smoking}

219 Smoking history and present smoking status were inquired about by way of two questions 220 at the age of 46 years: 1) "Have you ever smoked (yes/no)?" and 2) "Are you currently 221 smoking (yes/no)?" According to the answers we identified current and life-long 222 nonsmokers.

223 Alcohol use

224 The subjects were also asked if they used alcohol, and if so, what kinds, how often and 225 how much? Daily alcohol consumption was calculated according to the answers and 226 classified three ways: 1) never, 2) light 3) moderate or heavy use (women >20g/day).

\section{Education}

228 Education was classified into three groups by the number of years of education: 9 years, 9-12 years and more than 12 years. 


\section{Anxiety and Depressive symptoms}

Anxiety and depressive symptoms were assessed via the 25-item Hopkins Symptom Checklist (HSCL-25) at 46 years of age ${ }^{22,35}$. HSCL-25 part I includes 10 items concerning anxiety symptoms and part II, 15 items concerning depression. The scale varies between 1 and $4: 1=$ not troublesome to $4=$ extremely troublesome. The commonly used cut-off point of 1.55 was used to pinpoint anxiety and depression symptoms ${ }^{35}$.

\section{Statistical analyses}

A Tobit regression model ${ }^{33}$ was used to evaluate independent associations between endometriosis and PPT/MaxPTo. The motivation behind this was the large amount of censoring seen at the maximum limit of $1200 \mathrm{kPa}$. The interpretation of regression coefficients depends on the probability of not being censored. The interpretation is a combination of 1) the change in outcome, given that it is not censored, weighted by the probability of not being censored; and 2) the change in the probability of not being censored, weighted by the expected outcome if uncensored. Models were adjusted for BMI, anxiety and depression symptoms, smoking and contraceptive use.

Chi-squared tests were used to analyze the associations between the distribution and numbers of pain sites, and ANOVA was used to investigate the effect of pain intensity and troublesomeness at work, during leisure time and sleep. The analyses were performed with $\mathrm{R}$ software version 3.2.2, using the AER package for Tobit regression ${ }^{19}$.

\section{Results}

The prevalence of self-reported endometriosis was $8 \%$ and verification of the diagnosis was carried out by examining the hospital records (Supplemental Figure). Table 1 shows the characteristics of the study women and the controls. Of note is the fact that in the selfreported endometriosis group there was a relatively high percentage of women also having 
256 a diagnosis of endometriosis according to the hospital discharge register. The women with 257 self-reported endometriosis were more often nulliparous and suffering from infertility, 258 compared with controls. Use of hormonal contraceptives at any time was more frequent in women with endometriosis. No statistically significant differences were observed between the groups in terms of BMI, smoking, alcohol use or education level (Table 1).

The distribution of pain perception in women according to different conditions/confounders at age 46 is shown in Figure 2. Self-reported endometriosis was associated with statistically significant decreases in both pressure-pain threshold $(p<0.05$, Fig. 2A) and maximal pain tolerance $(p<0.001$, Fig. 2B) and the decreases in these variables remained after adjusting for different confounders. Other contributing factors were depression, anxiety and smoking. Interestingly, BMI and contraceptive use at any time seemed to increase the pain thresholds (Fig 2).

In Tobit regression analysis, PPT measurement showed that the women with endometriosis had on average a $34.0 \mathrm{kPa}$ lower $(-5.3 \%[-1.1,-9.5])$ pain threshold compared with controls $(p<0.05)$. As for the measurement site, PPT measured at the wrist was significantly lower in women reporting endometriosis $(-37.5 \mathrm{kPa}, \mathrm{p}<0.05$, Table 2), whereas the results concerning other measurement sites (shoulder, lower back and leg) 273 did not differ between the study groups. After adjusting for confounders, PPT remained 35.4 $\mathrm{kPa}$ lower in the endometriosis group $(\mathrm{p}<0.01)$. There were no statistically significant effects of BMI, anxiety, smoking or current or previous contraceptive use on pain threshold measured at the wrist.

Maximal pain tolerance was on average $-48.2 \mathrm{kPa}$ lower $(-5.1 \%[-2.2,-8.1])$ 278 among women with endometriosis $(p<0.001$, Table 2$)$ the change being significant at all 279 measurement sites, even after adjusting for BMI, anxiety and depressive symptoms, 280 smoking and contraceptive use (mean $-51.2 \mathrm{kPa}, \mathrm{p}<0.001)$, wrist $(-58.2 \mathrm{kPa})$, shoulder (- 
$28153.4 \mathrm{kPa})$, lower back (-58.0 kPa) and leg (-46.8 kPa).The most significant contributors 282 besides endometriosis that lowered maximum pain tolerance were anxiety, depression and 283 current smoking status $(-29.7 \mathrm{kPa},-28.5 \mathrm{kPa},-34.2 \mathrm{kPa}$, respectively) $(\mathrm{p}<0.05$, Figure 2$)$.

1, 2, 3, 4, 5-8 sites), pain troublesomeness and pain intensity (Fig. 3). Among women with endometriosis there were significantly fewer reporting no pain sites $(9.6 \%$ vs. $17.9 \%$, p<0.001, Fig. 3). Overall, the women with endometriosis also reported more pain sites compared with controls ( 1 site $17.4 \%$ vs. $16.2 \%, 2$ sites $17.0 \%$ vs $18.5 \%, 3$ sites $15.5 \%$ vs. $16.2 \%, 4$ sites $15.6 \%$ vs. $12.2 \%$ and $5-8$ sites $24,8 \%$ vs $19,1 \%, p<0.001$, Fig. 3 ).

As for pain troublesomeness, endometriosis was associated with slightly more troublesome pain at work and during leisure time and sleep $(p=0.01, p=0.02, p=0.04$ respectively, Fig. 4A). After adjusting pain troublesomeness for smoking, BMI, depression, anxiety and contraceptive use it was still significant during work $(p=0.04)$ whereas the significance was abolished for pain troublesomeness during leisure time and sleep $(p=0.05, p=0.06$, Fig. 4A). Adjusted overall pain intensity was also greater among women with endometriosis vs. controls ( $p=0.03$, Fig. 4B).

\section{Discussion}

299 This is the first population-based study to show an altered musculoskeletal pain response 300 and increased self-reported pain sensitivity, troublesomeness and intensity among women 301 at late reproductive age with a history of endometriosis. The results indicate that 302 endometriosis may have long-term consequences related to pain perception even at late 303 reproductive years.

Our data show a lower pressure-pain threshold and lower maximal pain 305 tolerance among 46-year-old women with a history of endometriosis compared with 
controls in a population-based study setting. The data adds to the body of evidence in the literature showing altered pain sensitivity in endometriosis. However, previous studies have been hospital-related populations ${ }^{1,2,4,12}$. In the present study, regression analysis suggested that endometriosis is associated independently with lower pain threshold and tolerance, whereas the strongest factors further decreasing the pain threshold and the maximal pain response were anxiety, depression and current smoking status. Given all this, it is worth noting that depression has been previously shown to be associated with altered pain perception ${ }^{15}$ and interestingly, musculoskeletal pain responses are particularly increased among women with co-expressing endometriosis and anxiety or depression symptoms ${ }^{31}$. Interestingly, both past and current contraceptive use appeared to be associated with unchanged pain tolerance, supporting the clinical use of hormonal contraceptives also in women with pelvic pain. The role of estrogens in pain perception is, however, complex. Interestingly, low estrogen concentrations in late menstrual cycle or during menopause or increased estrogen-testosterone ratio in male to female transsexuals have been shown to associate with increased pain symptoms ${ }^{38}$. Whether menopause solves the endometriosis-related altered pain responses or make them worse remains to be evaluated in future studies as estrogen measurements or menopausal status were not available for the present study.

As for individual pain-measurement sites, the pain threshold measured at the wrist in women with endometriosis was significantly lower compared with that in the controls. A similar trend was also shown at other pain-measurement sites. To our knowledge, ours is the first population based study to show decreased MaxPTo at several measurement sites among women of late reproductive age with a history of endometriosis, compared with controls and the results are in line with several previous studies also showing altered pain responses in women with endometriosis. In a previous study in which 
331 pressure-pain sensitivity was assessed in the thumbnail, the results showed significantly

332 lower pain threshold among women with symptomatic endometriosis ${ }^{2}$. Visceral

333 hypersensitivity testing also revealed lower pain thresholds among women with

334 endometriosis in a rectal balloon dilation test ${ }^{18}$ and lower pain thresholds and larger pain

335 areas were reported in women with symptomatic endometriosis after an intramuscular

336 saline injection into the hand ${ }^{4}$. In a more recent study concerning pressure-pain

337 thresholds at 20 different body sites, with use of the visual analog scale, it was reported

338 that there was a lower pain threshold in the greater trochanter and abdomen in fertile-aged

339 women with endometriosis compared with controls ${ }^{24}$. A population-based study carried

340 out by Slater et al., with similar musculoskeletal pain response testing as in the present

341 study, showed decreased pain thresholds in women experiencing severe menstrual pain ${ }^{29}$.

342 Given that about $70 \%$ of women with dysmenorrhea (CPP) present with endometriosis in

343 laparoscopy, the data by Slater et al. are in line with the present results underlying altered

344 pain perception in affected women. The mechanisms behind the lowered threshold are

345 most likely multifactorial, involving peripheral and central mechanisms ${ }^{23}$. Whether the

346 women with CPP are the ones who also have altered pain perception during late

347 reproductive years remains to be investigated as the present data did not record 348 dysmenorrhea or pelvic pain.

Women with endometriosis reported more pain sites, and graded pain to be

350 more bothersome and intense compared with controls. This might be due to central and/or

351 peripheral sensitization which has been shown to result from prolonged noxious pain 352 stimulation sustaining central pain stimulation in these cases ${ }^{17,31}$. Indeed, women with 353 endometriosis have reported increased regional hyperalgesia and allodynia ${ }^{31}$. Moreover, 354 the fact that pelvic pain correlates poorly with findings/severity of endometriosis further 355 emphasizes the fact that central and/or peripheral sensitization is most likely involved in 
356 the pain-regulatory system among affected women ${ }^{3,31}$. All in all, delayed diagnosis and

357 prolonged pain sensations may bring about altered pain sensitization among women with 358 endometriosis.

There are several strengths but also some limitations in the present work.

360 This is the first population-based study carried out to investigate pain perception/sensitivity 361 related to a history of endometriosis in women of late reproductive age. Women with 362 endometriosis were identified from a unique, large population-based data set of 363 homogeneous ethnicity and age and with the possibility to adjust for several confounding 364 factors. The data included objective pain measurements as well as subjective 365 questionnaire data. Moreover, the data collection did not specifically target endometriosis 366 patients or patients only treated in hospitals. Hence, the questionnaires and clinical 367 measurements were carried out in the whole cohort, with minimal self-aware bias. The 368 study also has limitations, which include self-reported endometriosis diagnosis and lack of 369 data on clinical symptoms of endometriosis; thus it is possible that the control group also 370 included women with endometriosis, albeit with milder pain symptoms/sensitivity. 371 However, the control group in the present data set was fairly large and such cases would 372 have been diluted among the controls. Moreover, studies on endometriosis commonly 373 concern only laparoscopically verified cases, and thus women with endometriosis with 374 fewer pain symptoms are most likely underrepresented in these studies. The self-reported 375 diagnoses of endometriosis may also be considered as a limitation, although, the 376 diagnosis was validated from the patient records available and from the national hospital 377 discharge register. In a recent study by Saha et al, similar results were presented when 378 self-reported endometriosis diagnoses were verified from patient records ${ }^{26}$. This was 379 further supported by a recent study validating self-reported endometriosis diagnosis in a 380 Swedish national twin registry ${ }^{27}$. The authors concluded that self-reported diagnosis 
381 seems to be moderately accurate, and when additional information is also available the 382 accuracy is even better. It must be noted, however, that even though laparoscopy is the 383 gold standard in endometriosis diagnosis, in some milder cases the operation is not 384 justified and thus the diagnosis remains clinical. Although our measurements showed 385 statistically significant $5 \%$ decreases in pain threshold and maximal pain tolerance in 386 women with endometriosis, the clinical significance remains uncertain, although these women also self-reported more pain symptoms. Furthermore, the associations between endometriosis-related pain symptoms and other comorbid pain syndromes, menopause or estradiol levels were not investigated due to lack of available data, thus these aspects remain to be evaluated in future studies.

To conclude, this is the first population-based study showing a decreased pain threshold and a decreased maximal pain response among women of late fertile age with a history of endometriosis. The fact that the women also reported a higher number of pain sites, with a greater prevalence of troublesome and intense pain at age 46 underlines the fact that endometriosis may have a long-term footprint as regards pain perception in these women. Given all this, women with endometriosis symptoms should be screened and 397 diagnosed as early as possible by a multidisciplinary team in order to ensure minimal comorbidity, adequate pain relief and psychological support. Further studies are warranted to address the diagnostic difficulties and different endometriosis phenotypes and also to 400 elucidate the pain mechanisms and best treatment options for these women. 
406 Acknowledgements:

407 We thank Tuula Ylitalo for assisting with data acquisition. We thank Dr. Nick Bolton for 408 correcting English. 


\section{References}

1. As-Sanie S, Harris RE, Napadow V, Kim J, Neshewat G, Kairys A, Williams D, Clauw DJ Schmidt-Wilcke T: Changes in regional gray matter volume in women with chronic pelvic pain: a voxel-based morphometry study. Pain 153 5:1006-1014, 2012

2. As-Sanie S, Harris RE, Harte SE, Tu FF, Neshewat G Clauw DJ: Increased pressure pain sensitivity in women with chronic pelvic pain. Obstet Gynecol 122 5:1047-1055, 2013

3. As-Sanie S, Kim J, Schmidt-Wilcke T, Sundgren PC, Clauw DJ, Napadow V Harris RE: Functional Connectivity is Associated With Altered Brain Chemistry in Women With Endometriosis-Associated Chronic Pelvic Pain. J Pain 17 1:1-13, 2016

4. Bajaj $P$, Bajaj $P$, Madsen $H$ Arendt-Nielsen L: Endometriosis is associated with central sensitization: a psychophysical controlled study. J Pain 4 7:372-380, 2003

5. Brawn J, Morotti M, Zondervan KT, Becker CM Vincent K: Central changes associated with chronic pelvic pain and endometriosis. Hum Reprod Update 20 5:737-747, 2014

6. Burney RO Giudice LC: Pathogenesis and pathophysiology of endometriosis. Fertil Steril 98 3:511-519, 2012

7. Dunselman GA, Vermeulen N, Becker C, Calhaz-Jorge C, D'Hooghe T, De Bie B, Heikinheimo O, Horne AW, Kiesel L, Nap A, Prentice A, Saridogan E, Soriano D, Nelen W European Society of Human Reproduction and Embryology: ESHRE guideline: management of women with endometriosis. Hum Reprod 29 3:400-412, 2014

8. Facchin F, Barbara G, Saita E, Mosconi P, Roberto A, Fedele L Vercellini P: Impact of endometriosis on quality of life and mental health: pelvic pain makes the difference. $J$ Psychosom Obstet Gynaecol 36 4:135-141, 2015

9. Fauconnier A Chapron C: Endometriosis and pelvic pain: epidemiological evidence of the relationship and implications. Hum Reprod Update 11 6:595-606, 2005

10. Fraser IS: Mysteries of endometriosis pain: Chien-Tien Hsu Memorial Lecture 2009. J Obstet Gynaecol Res 36 1:1-10, 2010

11. Gao X, Outley J, Botteman M, Spalding J, Simon JA Pashos CL: Economic burden of endometriosis. Fertil Steril 86 6:1561-1572, 2006

12. Giamberardino MA, Tana C Costantini R: Pain thresholds in women with chronic pelvic pain. Curr Opin Obstet Gynecol 26 4:253-259, 2014

\section{Giudice LC Kao LC: Endometriosis. Lancet 364 9447:1789-1799, 2004}

14. Gruppo Italiano per lo Studio dell'Endometriosi: Relationship between stage, site and morphological characteristics of pelvic endometriosis and pain. Hum Reprod 16 12:26682671, 2001 
15. Hermesdorf M, Berger K, Baune BT, Wellmann J, Ruscheweyh R Wersching H: Pain Sensitivity in Patients With Major Depression: Differential Effect of Pain Sensitivity Measures, Somatic Cofactors, and Disease Characteristics. J Pain , 2016

16. Howard FM: Endometriosis and mechanisms of pelvic pain. J Minim Invasive Gynecol 16 5:540-550, 2009

17. Imamura M, Imamura ST, Kaziyama HH, Targino RA, Hsing WT, de Souza LP, Cutait MM, Fregni F Camanho GL: Impact of nervous system hyperalgesia on pain, disability, and quality of life in patients with knee osteoarthritis: a controlled analysis. Arthritis Rheum 59 10:1424-1431, 2008

18. Issa B, Onon TS, Agrawal A, Shekhar C, Morris J, Hamdy S Whorwell PJ: Visceral hypersensitivity in endometriosis: a new target for treatment? Gut 61 3:367-372, 2012

19. Kleiber C, Zeileis A SpringerLink: Applied Econometrics with R. , 2008

20. Kucyi A, Salomons TV Davis KD: Cognitive behavioral training reverses the effect of pain exposure on brain-network activity. Pain , 2016

21. Laufer MR, Goitein L, Bush M, Cramer DW Emans SJ: Prevalence of endometriosis in adolescent girls with chronic pelvic pain not responding to conventional therapy. J Pediatr Adolesc Gynecol 10 4:199-202, 1997

22. Mattisson C, Bogren M Horstmann V: Correspondence between clinical diagnoses of depressive and anxiety disorders and diagnostic screening via the Hopkins Symptom Check List-25 in the Lundby Study. Nord J Psychiatry 67 3:204-213, 2013

23. Morotti M, Vincent K Becker CM: Mechanisms of pain in endometriosis. Eur J Obstet Gynecol Reprod Biol , 2016

24. Nunes FR, Ferreira JM Bahamondes L: Pain threshold and sleep quality in women with endometriosis. Eur J Pain 19 1:15-20, 2015

25. Porpora MG, Koninckx PR, Piazze J, Natili M, Colagrande S Cosmi EV: Correlation between endometriosis and pelvic pain. J Am Assoc Gynecol Laparosc 6 4:429-434, 1999

26. Saha R, Kuja-Halkola R, Tornvall P Marions L: Reproductive and Lifestyle Factors Associated with Endometriosis in a Large Cross-Sectional Population Sample. J Womens Health (Larchmt) , 2016

27. Saha R, Marions L Tornvall $P$ : Validity of self-reported endometriosis and endometriosis-related questions in a Swedish female twin cohort. Fertil Steril 107 1:174178.e2, 2017

28. Sinaii N, Cleary SD, Ballweg ML, Nieman LK Stratton P: High rates of autoimmune and endocrine disorders, fibromyalgia, chronic fatigue syndrome and atopic diseases among women with endometriosis: a survey analysis. Hum Reprod 17 10:2715-2724, 2002 
29. Slater H, Paananen M, Smith AJ, O'Sullivan P, Briggs AM, Hickey M, Mountain J, Karppinen J Beales D: Heightened cold pain and pressure pain sensitivity in young female adults with moderate-to-severe menstrual pain. Pain 156 12:2468-2478, 2015

30. Soliman AM, Yang H, Du EX, Kelley C Winkel C: The direct and indirect costs associated with endometriosis: a systematic literature review. Hum Reprod 31 4:712-722, 2016

31. Stratton P, Khachikyan I, Sinaii N, Ortiz R Shah J: Association of chronic pelvic pain and endometriosis with signs of sensitization and myofascial pain. Obstet Gynecol 125 $3: 719-728,2015$

32. Tarjanne S, Ng CH, Manconi F, Arola J, Mentula M, Maneck B, Fraser IS Heikinheimo $\mathrm{O}$ : Use of hormonal therapy is associated with reduced nerve fiber density in deep infiltrating, rectovaginal endometriosis. Acta Obstet Gynecol Scand 94 7:693-700, 2015

33. Tobin J: Estimation of Relationships for Limited Dependent Variables. Econometrica 26 1:24-36, 1958

34. Triolo O, Lagana AS Sturlese E: Chronic pelvic pain in endometriosis: an overview. J Clin Med Res 5 3:153-163, 2013

35. Veijola J, Jokelainen J, Laksy K, Kantojarvi L, Kokkonen P, Jarvelin MR Joukamaa M: The Hopkins Symptom Checklist-25 in screening DSM-III-R axis-I disorders. Nord J Psychiatry 57 2:119-123, 2003

36. Vercellini P, Trespidi L, De Giorgi O, Cortesi I, Parazzini F Crosignani PG:

Endometriosis and pelvic pain: relation to disease stage and localization. Fertil Steril 65 2:299-304, 1996

37. Vercellini P, Vigano P, Somigliana E Fedele L: Endometriosis: pathogenesis and treatment. Nat Rev Endocrinol 10 5:261-275, 2014

38. Vincent K Tracey I: Hormones and their Interaction with the Pain Experience. Rev Pain 2 2:20-24, 2008 
505 Figure 1. Flowchart showing the study population $\left(\mathrm{n}_{\text {endometriosis }}=284, \mathrm{n}_{\text {controls }}=3390\right)$

506 derived from Northern Finland Birth Cohort 1966.

Figure 2. Pain perception in women according to different conditions/confounders

509 at

510 age 46. The horizontal reference line reflects the whole study population. Self-reported

511 endometriosis appeared to result in decreases in both pressure pain threshold (PPT)

$512(p<0.05, A)$, and maximum pressure pain tolerance (MaxPTo) $(p<0.001, B)$ compared with 513 the effect of BMI and contraceptive use at any time.

515 Figure 3. The numbers of reported pain sites in women with endometriosis (black)

516 and in controls (gray) at age 46. Percentages of women experiencing $0,1,2,3,4$ or $5-8$

517 pain sites per year. Fewer women with endometriosis (black bars) reported having no pain 518 sites compared with controls (gray bars) $(p<0.001)$. The numbers of pain sites were 519 increased in women with endometriosis compared with controls $(p<0.001)$.

Figure 4. Pain troublesomeness $(A)$ and intensity $(B)$ in women with endometriosis and in controls at age 46. A) The mean pain troublesomeness score was increased in women with endometriosis (black bars) compared with controls (gray bars) at work. A similar trend was seen during leisure and sleep.

B) The women with endometriosis reported having more intense pain compared with controls. Mean numerical rating (MNR)

526 is the mean of pain scoring from 0 to 5. 
528 Suppelemental Figure. Validation of 284 self-reported endometriosis diagnosis was

529 carried out by going through patient records available (92 cases) at the original study site. 530 
Table 1. Patient characteristics in women with self-reported endometriosis and controls at age 46 according to questionnaire data.

\begin{tabular}{|c|c|c|c|}
\hline 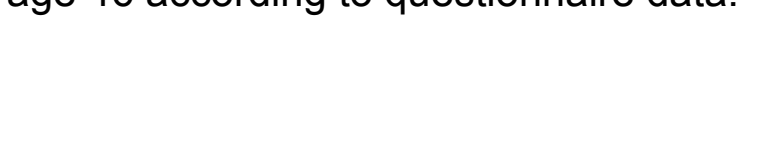 & $\begin{array}{l}\text { Endometriosis } \\
\qquad(\mathrm{n}=284)^{\star} \\
\%\end{array}$ & $\begin{array}{l}\text { Controls } \\
(\mathrm{n}=3390)^{*} \\
\%\end{array}$ & p-value \\
\hline $\begin{array}{l}\text { Endometriosis diagnosis in the national } \\
\text { hospital discharge registry }\end{array}$ & 52.0 & 1.5 & \\
\hline Suffering from infertility & 33.8 & 14.1 & $<0.001$ \\
\hline No delivery & 13.9 & 9.8 & \multirow{3}{*}{$<0.001$} \\
\hline One delivery & 24.2 & 16.2 & \\
\hline More than one delivery & 61.9 & 73.9 & \\
\hline $\begin{array}{l}\text { Use of hormonal contraceptives } \\
\text { Ever } \\
\text { Current }\end{array}$ & $\begin{array}{l}93.3 \\
20.2\end{array}$ & $\begin{array}{l}89.2 \\
27.1\end{array}$ & $\begin{array}{l}0.033 \\
0.028\end{array}$ \\
\hline $\begin{array}{l}\text { BMI }(\mathrm{kg} / \mathrm{m} 2) \\
<18.5 \\
18.5-24.999 \\
25-29.999 \\
\geq 30\end{array}$ & $\begin{array}{c}0.8 \\
48.5 \\
32.8 \\
17.8\end{array}$ & $\begin{array}{c}0.9 \\
45.4 \\
32.8 \\
20.9\end{array}$ & 0.676 \\
\hline $\begin{array}{l}\text { Smoking } \\
\text { Ever } \\
\text { Current }\end{array}$ & $\begin{array}{l}51.4 \\
33.6\end{array}$ & $\begin{array}{l}52.3 \\
32.6\end{array}$ & 0.804 \\
\hline \multicolumn{4}{|l|}{ Alcohol use } \\
\hline Never & 6.0 & 6.2 & \multirow{3}{*}{0.603} \\
\hline Light (less than monthly) & 11.7 & 11.7 & \\
\hline Moderate/heavy (at least once in a month) & 76.3 & 77.7 & \\
\hline $\begin{array}{l}\text { Education } \\
\text { Basic } \\
\text { Secondary } \\
\text { Tertiary }\end{array}$ & $\begin{array}{l}1.8 \\
50.7 \\
47.5\end{array}$ & $\begin{array}{l}2.3 \\
57.1 \\
40.6\end{array}$ & 0.072 \\
\hline
\end{tabular}

${ }^{*} \mathrm{n}$ varies in some of the variables due to missing questionnaire data 
Table 2. Tobit regression analysis of pressure pain threshold (PPT) and maximal pain tolerance (MaxPTo) in women with endometriosis compared with controls

\begin{tabular}{|c|c|c|c|c|c|}
\hline & \multirow[b]{2}{*}{ Average } & \multicolumn{4}{|c|}{ Location of pressure pain measurement } \\
\hline & & Wrist & Shoulder & Lower back & Leg \\
\hline & kPa $(95 \% \mathrm{Cl})$ & $\mathrm{kPa}(95 \% \mathrm{Cl})$ & kPa $(95 \% \mathrm{Cl})$ & $\mathrm{kPa}(95 \% \mathrm{Cl})$ & $\mathrm{kPa}(95 \% \mathrm{Cl})$ \\
\hline Observations (total) & $n=2609$ & $n=2730$ & $\mathrm{n}=2747$ & $\mathrm{n}=2635$ & $n=2738$ \\
\hline${ }^{\#}$ Constant PPT (crude) & $642.6(634.2,650.9)$ & $648.8(639.6,657.9)$ & $585.8(575.8,595.8)$ & $710.2(699.1,721.3)$ & $641.1(630.7,651.5)$ \\
\hline \multirow{2}{*}{$\begin{array}{l}\text { Endometriosis PPT (crude) } \\
\wedge \text { Difference [\%] }\end{array}$} & $-34.0^{*}(-60.8,-7.3)$ & $-37.5^{\star}(-67.3,-7.7)$ & $-27.8(-58.9,3.2)$ & $-26.9(-63.0,9.1)$ & $-29.9(-63.5,3.7)$ \\
\hline & $-5.3 \%(-1.1,-9.5)$ & $-5.8 \%(-1.2,-10.4)$ & $-4.8 \%(0.5,-10.1)$ & $-3.8 \%(1.3,-8.9)$ & $-4.7 \%(0.6,-9.9)$ \\
\hline Constant PPT (adjusted) ${ }^{\star \star}$ & $645.7(620.9,670.6)$ & $648.6(620.8,676.4)$ & $602.1(572.3,631.9)$ & $690.8(657.4,724.1)$ & $649.1(617.3,680.8)$ \\
\hline Endometriosis PPT (adjusted) ${ }^{\star *}$ & $-35.4^{\star}(-62.2,-8.6)$ & $-36.4^{*}(-66.3,-6.6)$ & $-25.7(-56.5,5.0)$ & $-33.8(-69.5,2.0)$ & $-31.3(-65.0,2.3)$ \\
\hline${ }^{\wedge}$ Difference [\%] & $-5.5 \%(-1.3,-9.6)$ & $-5.6 \%(-1.0,-10.2)$ & $-4.3 \%(0.8,-9.4)$ & $-4.9 \%(0.3,-10.1)$ & $-4.8 \%(0.4,-10.0)$ \\
\hline Constant MaxPTo (crude) & $939.9(932.0,947.9)$ & $932.2(922.5,941.8)$ & $957.8(946.2,969.4)$ & $1031.3(1018.9,1043.8)$ & $941.4(989.1,1069.1)$ \\
\hline Endometriosis MaxPTo (crude) & $-48.2^{*}(-76.1,-20.4)$ & $-58.1^{\star}(-90.6,-25.6)$ & $-55.4^{*}(-93.1,-17.7)$ & $-50.6^{\star}(-91.0,-10.2)$ & $-43.7^{\star}(-81.0,-6.3)$ \\
\hline${ }^{\wedge}$ Difference $[\%]$ & $-5.1 \%(-2.2,-8.1)$ & $-6.2 \%(-2.7,-9.7)$ & $-5.8 \%(-1.8,-9.7)$ & $-4.9 \%(-1.0,-8.8)$ & $-4.6 \%(-0.7,-8.6)$ \\
\hline Constant MaxPTo (adjusted) ${ }^{* *}$ & $952.7(928.2,977.3)$ & $930.8(901.7,960.0)$ & $997.5(962.2,1032.7)$ & $1029.1(989.1,1069.1)$ & $953.2(917.4,989.0)$ \\
\hline Endometriosis MaxPTo (adjusted) ${ }^{\star *}$ & $-50.1^{*}(-78.0,-22.2)$ & $-58.2^{*}(-90.8,-25.6)$ & $-53.4^{*}(-90.7,-16.2)$ & $-58.0^{*}(-97.8,-18.1)$ & $-46.8^{*}(-84.2,-9.5)$ \\
\hline${ }^{\wedge}$ Difference [\%] & $-5.3 \%(-2.3,-8.2)$ & $-6.3 \%(-2.8,-9.8)$ & $-5.4 \%(-1.6,-9.1)$ & $-5.6 \%(-1.8,-9.5)$ & $-4.9 \%(-1.0,-8.8)$ \\
\hline
\end{tabular}

${ }^{* *}$ Adjusted for BMI, anxiety and depressive symptoms, smoking and use of hormonal contraceptives

\#Constant, a built estimate reference value for subjects with BMI at the mean level of the population, no significant anxiety or depressive symptoms, never smoker and no use of hormonal contraceptives

^Difference compared with controls 


\section{$9 \quad$ Figure 1}

10

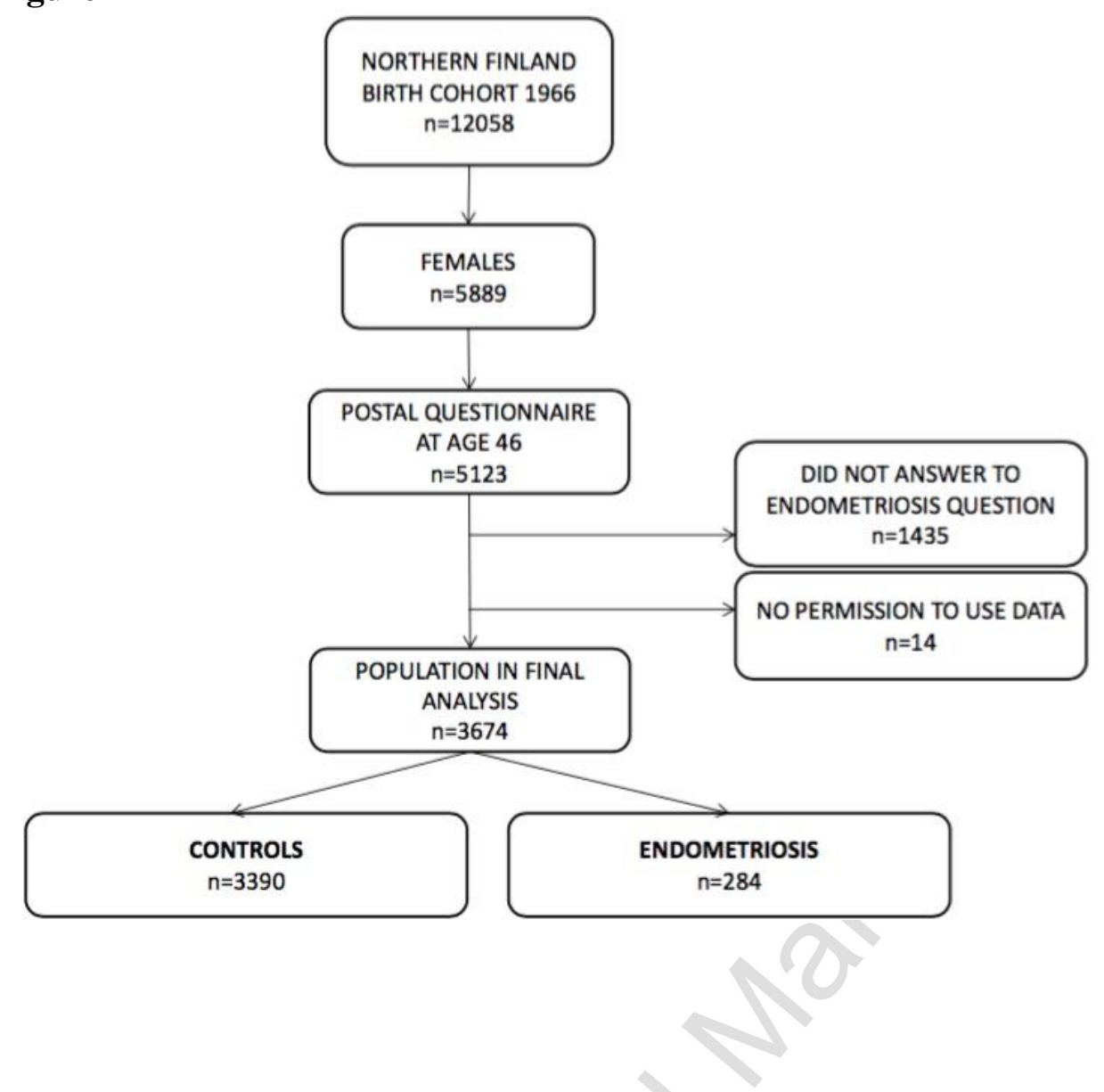




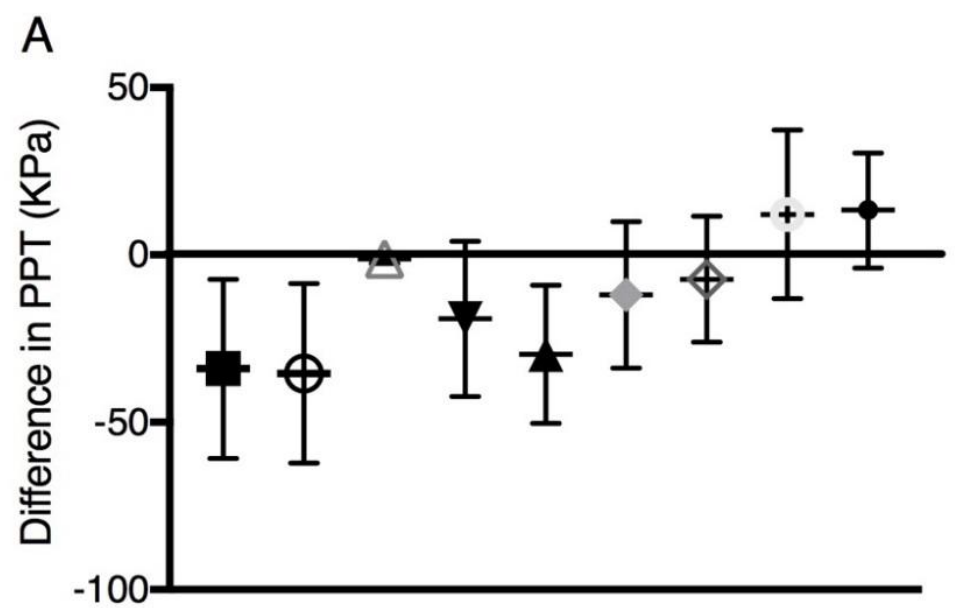

- Crude Endometriosis

O Adjusted Endometriosis

$\triangle \mathrm{BMl}$

$\nabla$ Anxiety $>1.55$ (HSCL)

$\Delta$ Depression $>1.55$ (HSCL)

Smoking (current)

$\diamond$ Smoking (former) Contraceptive use (ever)

- Contraceptive use (current)

15

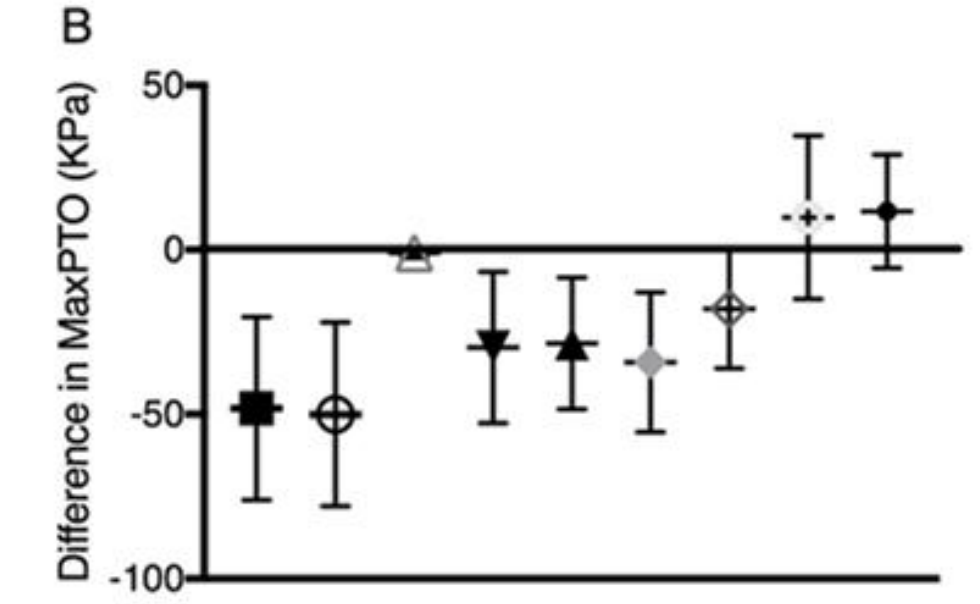

\section{Figure 2A}

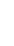

B

\section{Figure 2B}

- Crude Endometriosis

O Adjusted Endometriosis

$\triangle$ BMI

$\nabla$ Anxiety $>1.55$ (HSCL)

$\Delta$ Depression >1.55 (HSCL)

Smoking (current)

$\diamond$ Smoking (former) Contraceptive use (ever)

- Contraceptive use (current) 


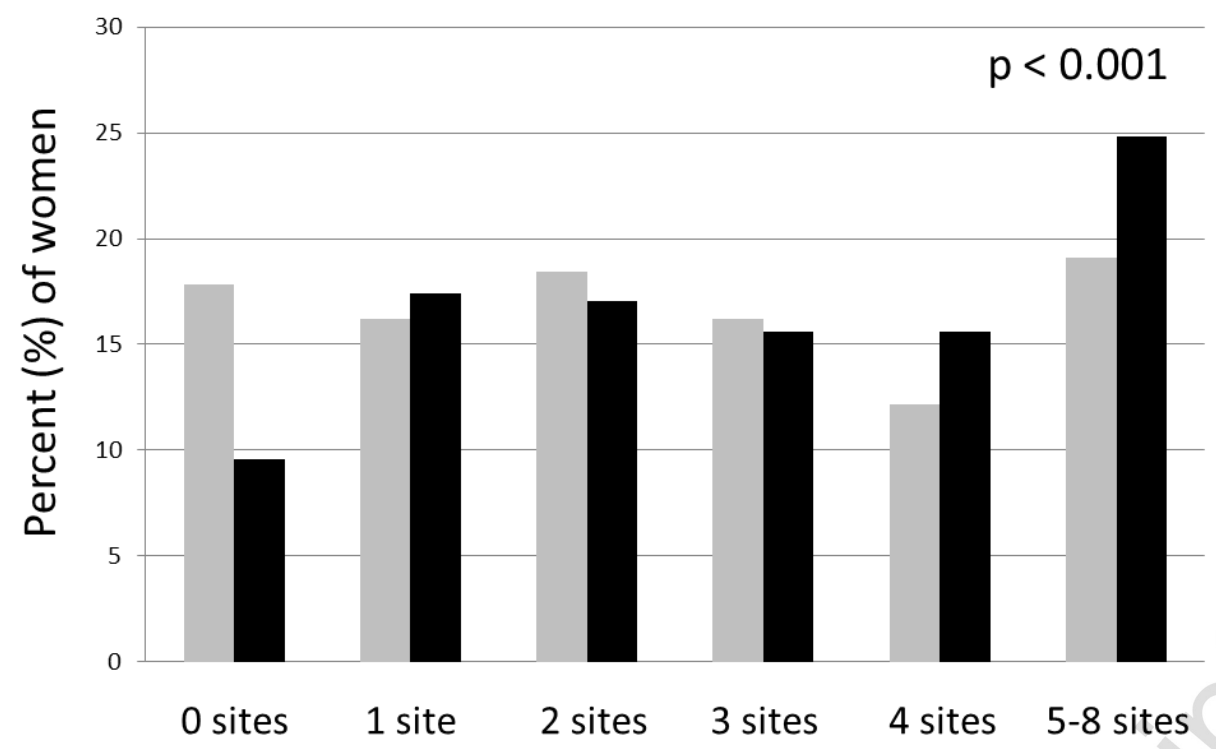

29

30

31

32

33

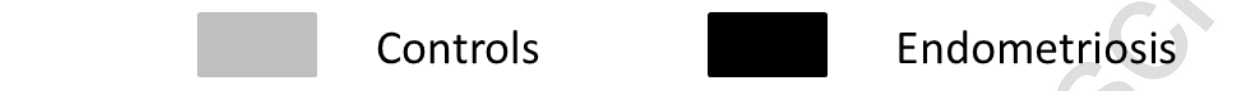

\section{Figure 3}

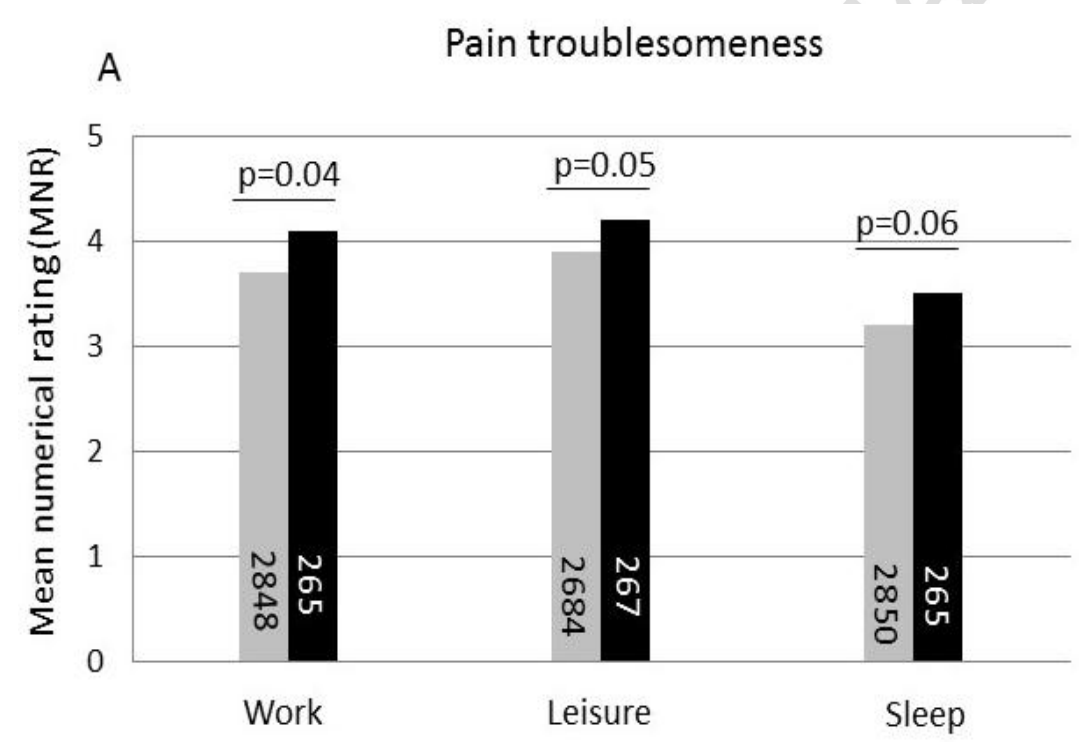

Controls
B Pain intensity

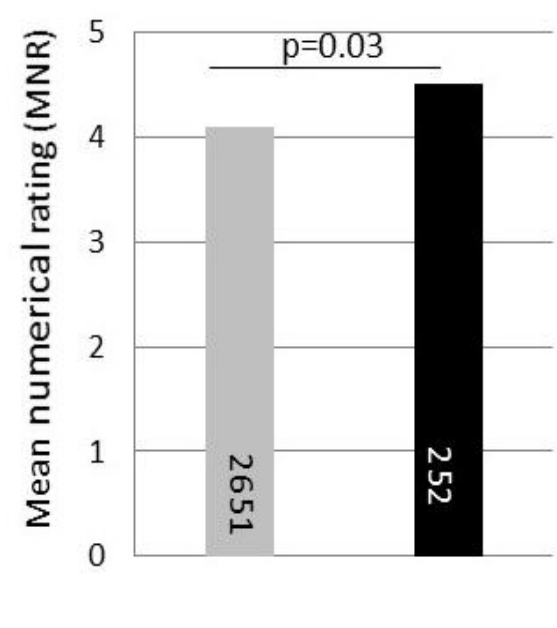

